\title{
Automated Characterization of Spent Fuel through the Multi- Isotope Process (MIP) Monitor
}

\author{
JB Coble \\ CR Orton \\ JM Schwantes
}

July 2012

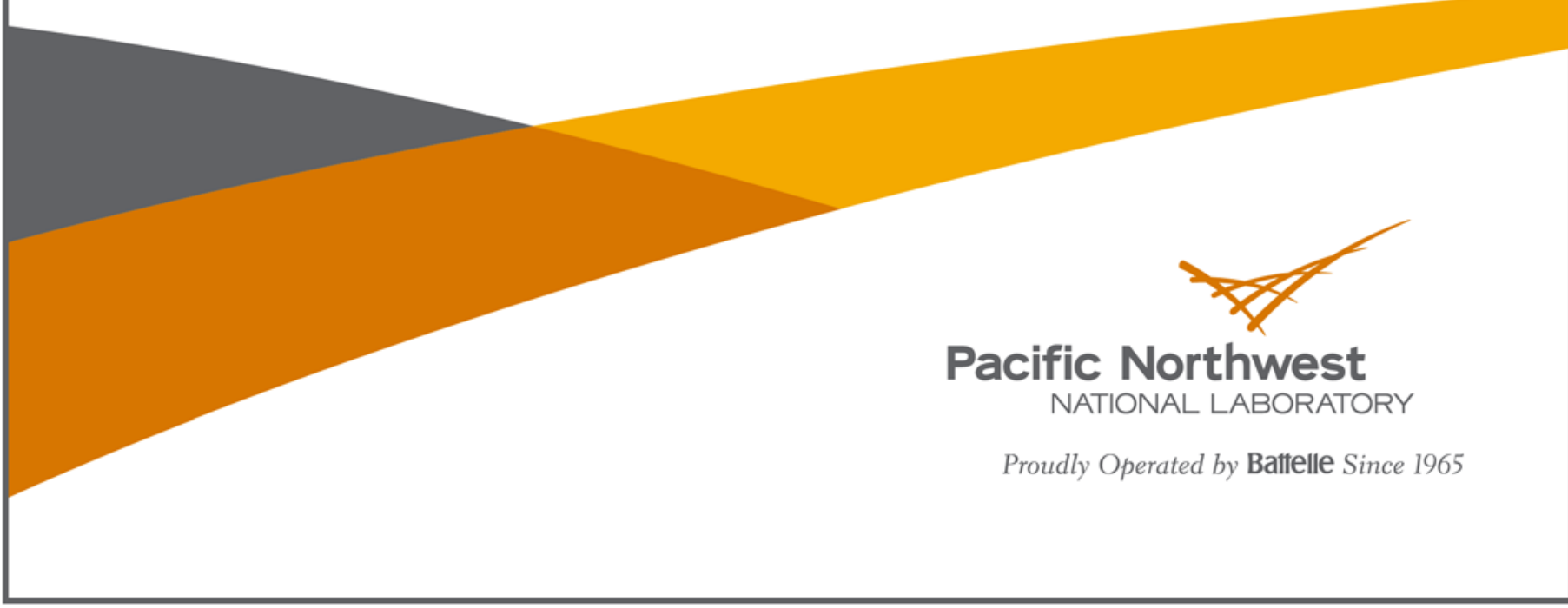




\title{
DISCLAIMER
}

This report was prepared as an account of work sponsored by an agency of the United States Government. Neither the United States Government nor any agency thereof, nor Battelle Memorial Institute, nor any of their employees, makes any warranty, express or implied, or assumes any legal liability or responsibility for the accu racy, completeness, or usefulness of any information, apparatus, product, or process disclosed, or represents that its use would not infringe privately owned rights. Reference herein to any specific commercial product, process, or service by trade name, trademark, manufacturer, or otherwise does not necessarily constitute or imply its endorsement, recommendation, or favoring by the United States Government or any agency thereof, or Battelle Memorial Institute. The views and opinions of authors expressed herein do not necessarily state or reflect those of the United States Government or any agency thereof.

\author{
PACIFIC NORTHWEST NATIONAL LABORATORY \\ operated by \\ BATTELLE \\ for the \\ UNITED STATES DEPARTMENT OF ENERGY \\ under Contract DE-AC05-76RL01830
}

Printed in the United States of America

Available to DOE and DOE contractors from the

Office of Scientific and Technical Information,

P.O. Box 62, Oak Ridge, TN 37831-0062;

ph: (865) 576-8401

fax: $(865) 576-5728$

email: reports@adonis.osti.gov

Available to the public from the National Technical Information Service

5301 Shawnee Rd., Alexandria, VA 22312

ph: (800) 553-NTIS (6847)

email: orders $a$ ntis.gov <http://www.ntis.gov/about/form.aspx>

Online ordering: http://www.ntis.gov

से This document was printed on recycled paper

(8/2010) 


\section{Automated Characterization of Spent Fuel through the Multi- Isotope Process (MIP) Monitor}

JB Coble

CR Orton

JM Schwantes

July 2012

Prepared for

the U.S. Department of Energy

under Contract DE-AC05-76RL01830

Pacific Northwest National Laboratory

Richland, Washington 99352 



\section{Summary}

The Multi-Isotope Process (MIP) Monitor provides an efficient approach to monitoring the process conditions in reprocessing facilities in support of the goal of "... (minimization of) the risks of nuclear proliferation and terrorism" (OTA 1995). The monitor utilizes multivariate analysis and gamma spectroscopy of reprocessing streams to detect small changes in the gamma spectrum, which may indicate changes in process conditions. Multivariate analysis methods common in chemometrics, such as principal component analysis (PCA) and partial least squares regression (PLS), act as pattern recognition techniques, which can detect small deviations from the expected, nominal condition. In past research, a database of training data covering "normal" conditions was used to train these multivariate methods (Orton et al. 2011, 2012). This approach can identify gross changes in process parameters, but small

deviations may be obfuscated by the range of training data. As the range of normal conditions included in training increases (e.g., by including fuel samples from many different reactors and operating conditions), the sensitivity of the model to small deviations may decrease. This research extends the MIP Monitor by characterizing the spent fuel samples after initial dissolution. By characterizing the spent fuel, multivariate models can be trained using only gamma spectra of fuel samples similar to the current one. This will improve the sensitivity of the MIP Monitor to very small changes at later stages in the process. Additionally, this automated fuel characterization can be used to verify spent fuel inventories unobtrusively, in near real time.

This research developed an algorithm for characterizing spent nuclear fuel (SNF) samples based on simulated gamma spectra. The gamma spectra for a variety of light water reactor fuels typical of those found in the United States were simulated. Fuel nuclide concentrations were simulated in ORIGEN-ARP for 1296 fuel samples with a variety of reactor designs, initial enrichments, burn ups, and cooling times. The results of the ORIGEN-ARP simulation were then input to SYNTH to simulate the gamma spectrum for each sample. These spectra were evaluated with PLS-based multivariate analysis methods to characterize the fuel according to reactor type (pressurized or boiling water reactor), enrichment, burn up, and cooling time. Characterizing some of the features in series by using previously estimated features in the prediction greatly improves the performance. By first classifying the spent fuel reactor type and then using type-specific models, the prediction error for enrichment, burn up, and cooling time improved by a factor of two to four. For some features, the prediction was further improved by including additional information, such as including the predicted burn up in the estimation of cooling time. The optimal prediction flow was determined based on the simulated data. A PLS discriminate analysis model was developed which perfectly classified SNF reactor type. Burn up was predicted within $0.1 \%$ root mean squared percent error (RMSPE) and both cooling time and initial enrichment within approximately $2 \%$ RMSPE.

The fuel characterization algorithm developed in this research used simulated data for model training and testing, which limits the applicability of the developed models. The simulated data suffers from the systematic errors in both simulation codes. Both codes have known inaccuracies based on the results of benchmarking. Additionally, neither of the simulators applied account for random noise, either process noise (e.g., normal changes in nuclide activity due to specific reactor operation) or measurement noise (e.g., counting noise due to the random nature of decay). Despite these limitations, this research suggests that similar analyses can be performed on actual gamma spectra collected from dissolved fuel samples. The concepts and framework developed are generically applicable, though the specific models and prediction flow order used will need to be developed and trained on actual data, either experimental or operational. 



\section{Acronyms and Abbreviations}

$\begin{array}{ll}\text { BWR } & \text { boiling water reactor } \\ \text { DA } & \text { destructive assay } \\ \text { E/F ratio } & \text { extract to aqueous feed phase volume ratio } \\ \text { GUI } & \text { graphical user interface } \\ \text { HPGe } & \text { high-purity germanium } \\ \text { iPLS } & \text { interval-PLS } \\ \text { LaBr } & \text { lanthanum bromide } \\ \text { LV } & \text { latent variable } \\ \text { LWPLS } & \text { locally weighted PLS } \\ \text { MC\&A } & \text { material control and accountability } \\ \text { MIP } & \text { Multi-Isotope Process } \\ \text { NaI } & \text { sodium iodide } \\ \text { NRT } & \text { near-real-time } \\ \text { PC } & \text { principal component } \\ \text { PCA } & \text { principal component analysis } \\ \text { PLS } & \text { partial least squares regression } \\ \text { PLSDA } & \text { PLS discriminate analysis } \\ \text { PNNL } & \text { Pacific Northwest National Laboratory } \\ \text { PWR } & \text { pressurized water reactor } \\ \text { RMSPE } & \text { root mean squared percent error } \\ \text { SNF } & \text { spent nuclear fuel } \\ \text { SNM } & \text { special nuclear materials } \\ \text { SNR } & \text { signal-to-noise ratio } \\ \text { TIMS } & \text { thermal ionization mass spectrometry } \\ & \end{array}$





\section{Contents}

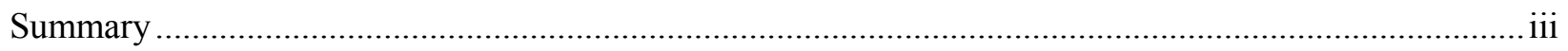

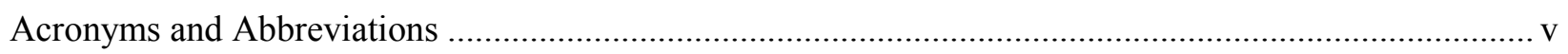

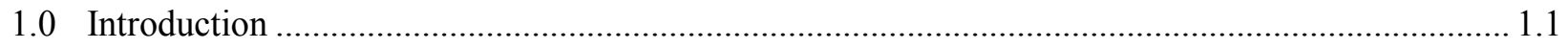

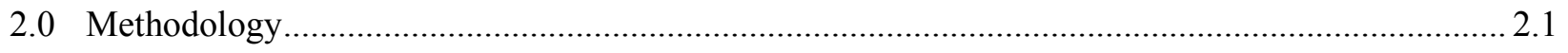

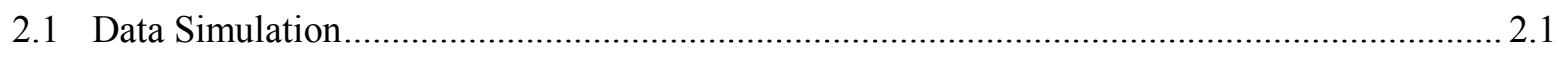

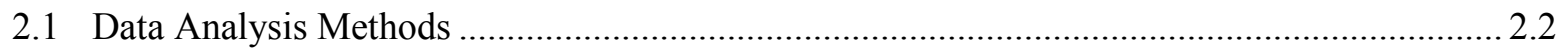

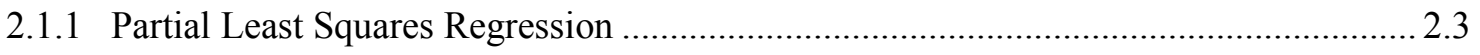

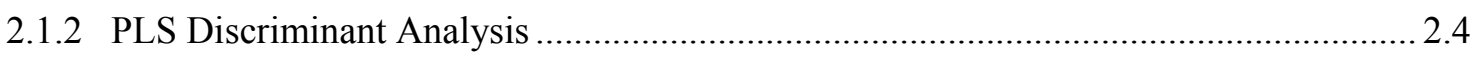

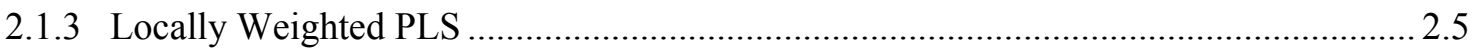

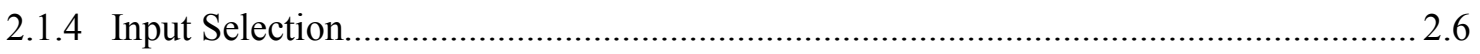

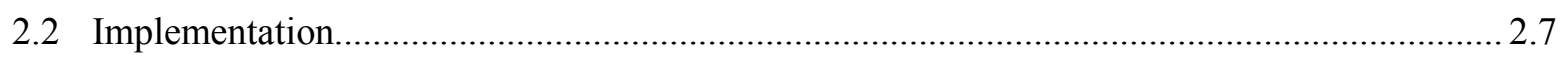

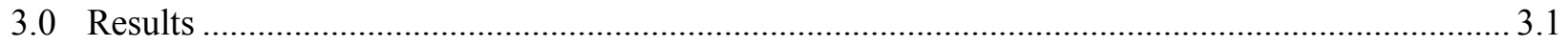

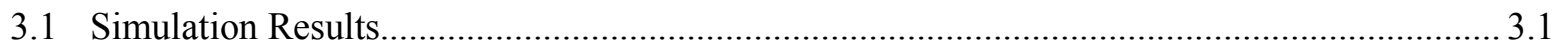

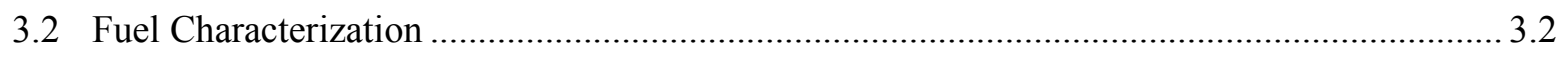

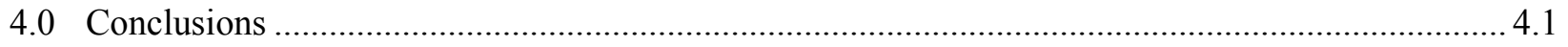

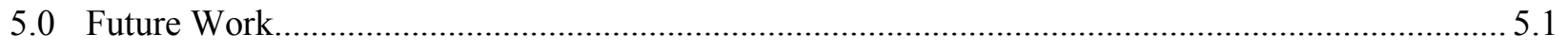

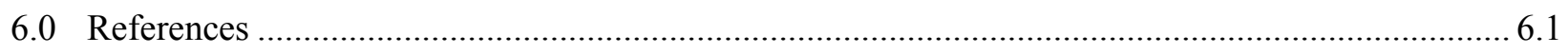

Appendix A Simulated Nuclides from ORIGIN-ARP ..................................................................... A.1

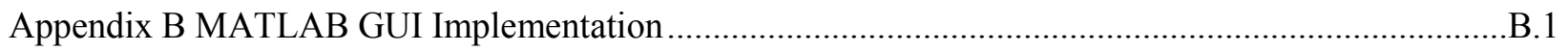




\section{Figures}

2.1 Fuel Characterization GUI (a) Before Spectra are Loaded and (b) After Spectra are Evaluated

3.1 Simulated Gamma Spectra for 1296 Example Spent Fuel Samples ............................................. 3.1

3.2 Selected Channels for Reactor Type Classification with PLSDA ….............................................. 3.3

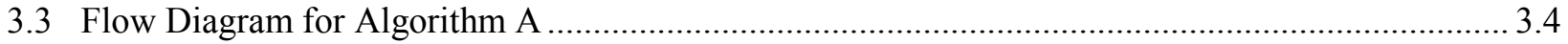

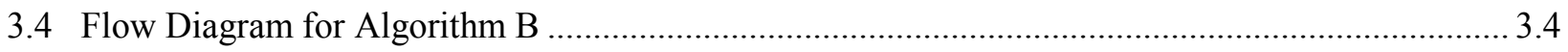

\section{Tables}

2.1 Spent Fuel Characteristics Used for Data Simulation............................................................ 2.1

2.2 HPGe Detector and Electronics Parameters for SYNTH Gamma Spectra Simulations ................... 2.2

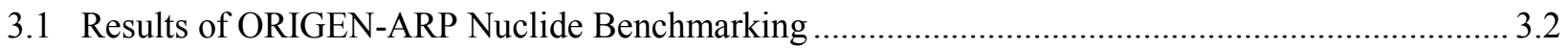

3.2 Key Gamma Contributors for Reactor Type Classification........................................................ 3.4

3.3 Fuel Characterization RMSPE for Parallel Prediction, Algorithm A, and Algorithm B ................. 3.5 


\subsection{Introduction}

Material Control \& Accountability (MC\&A) programs at nuclear processing plants are set up to deter and detect theft and diversion of nuclear material by both outside and inside adversaries. Current accountancy techniques rely heavily on state-of-the-art thermal ionization mass spectrometry (TIMS) for analyses of special nuclear materials (SNM) within accountancy tanks. While highly accurate and precise, TIMS measurements require extensive preprocessing by skilled staff. In the time it takes to generate these analytical results, significant quantities of SNM could be diverted within a large reprocessing facility. In addition, errors associated with these results, when used for material balance purposes, scale with the size of the accountancy tank. Without augmenting current destructive assay (DA)-based MC\&A approaches, the propagation of such errors might ultimately limit the size of a material balance area or even the overall size of a reprocessing facility. However, on-line, near-real-time (NRT), process monitoring technologies, when applied in concert with traditional DA techniques, may have the ability to significantly reduce required resources and improve the timeliness of MC\&A at reprocessing facilities.

The Multi-Isotope Process (MIP) Monitor is being developed at Pacific Northwest National Laboratory (PNNL) as one approach to online process monitoring for MC\&A. The MIP Monitor measures distributions of a suite of indicator (radioactive) isotopes present within product and waste streams of a nuclear reprocessing facility. These indicator isotopes are monitored on-line by gamma spectrometry and compared, in near-real-time, to spectral patterns representing "normal" process conditions using multivariate pattern recognition software. By targeting multiple gamma-emitting indicator isotopes, the MIP Monitor approach is compatible with the use of small, portable, relatively high-resolution gamma detectors that may be easily deployed throughout an existing facility.

The MIP Monitor is designed to track plant conditions indirectly and non-destructively by monitoring numerous gamma-emitting radioactive constituents within process streams. The behavior of many fission and activation products within a reprocessing facility will be dictated by a number of operational variables including (but not limited to) acid concentration, TBP concentration, temperature, burn up, cooling time, etc. In these bi-phase systems, small amounts of both product and contaminants remain in the other phase. The distribution of each element between the organic and aqueous phases is determined by major process variables such as acid concentration, organic ligand concentration, organic extract to aqueous feed phase volume ratio (E/F ratio), reduction potential, and temperature. Hence, for a consistent industrial process (i.e., steady-state and reproducible conditions), the distribution of each element between the organic and aqueous phases should be relatively constant. Consequently, the pattern of elements distributing into product and waste streams at each stage in the facility should be relatively reproducible (within normal industrial variations) resulting in a signature indicative of "normal" process conditions. Under abnormal conditions (such as those expected under some protracted diversion scenarios), patterns of elements within the various streams would be expected to change in possibly small, but measureable, ways. The MIP monitoring approach capitalizes on these expected changes in the distribution patterns of gamma-emitting elements in the aqueous and organic phases to serve as an indication of "off-normal" conditions in the process chemistry. The MIP Monitor is designed to track numerous radioactive constituents within process streams to indirectly monitor conditions in that stream. The monitor relies on small, medium-resolution gamma detectors that do not require cryogenic cooling and that can be easily placed throughout an existing facility. Multivariate analysis is used to autonomously process the spectra from these detectors in NRT, continuously monitoring for indications in changing stream conditions. 
Initial simulations and experiments have documented the performance and potential of the MIP Monitor (Orton et al. 2011, 2012). These early efforts have focused on detecting changes in process conditions, such as acid concentration, from a set of nominal conditions.

The research presented in this report expands the MIP Monitor approach by characterizing the reactor type (i.e., pressurized or boiling water, PWR or BWR, respectively), initial enrichment, burn up, and cooling time of spent nuclear fuel (SNF) based on simulated gamma spectra. By characterizing and classifying the spent fuel at initial dissolution, we can (1) verify that the fuel is consistent with written inventories and (2) utilize fuel-specific multivariate models to monitor conditions later in the process. Using fuel-specific multivariate models will improve the sensitivity of the MIP Monitor to small perturbations and anomalies in the process.

The multivariate analysis methods explored in this research were applied to simulated data, which limits the applicability of the developed models. The simulated data suffers from the systematic errors in two levels of simulation - ORIGEN-ARP and SYNTH. ORIGEN-ARP is used to simulate the activities of nuclides present in a spent fuel sample of given reactor type, initial enrichment, burn up, and cooling time. The accuracy of the simulated activities of selected nuclides has been benchmarked for similar SNF (Gauld and Litwin 1995; Leal et al. 1999; Gauld and Murphy 2004); the pertinent results of benchmarking will be summarized. Additionally, SYNTH simulates gamma spectra based on the output of the ORIGEN-ARP simulations; additional errors may be introduced by this code. Neither of the simulators applied account for random noise, either process noise (e.g., normal changes in nuclide activity due to specific reactor operation) or measurement noise (e.g., counting noise due to the random nature of decay). Despite these limitations, the performance of the models developed in this research suggest that similar analyses can be performed on actual gamma spectra collected from dissolved fuel. The concepts and framework developed are generically applicable, though the specific models used will need to be developed and trained on actual, experimental, or operational data.

This report presents the multivariate analysis framework developed to characterize spent fuel based on gamma spectra. The following section presents the methodologies used for data simulation and data analysis. The MATLAB-based implementation is briefly described, as well as a graphical user interface (GUI)-based implementation. Section 3 presents the results of the data simulation and fuel characterization. Finally, conclusions and areas for future related research are given in Sections 4 and 5, respectively. 


\subsection{Methodology}

The MIP Monitor capitalizes on small changes in the gamma spectra from an expected, nominal spectrum to detect changes in the process conditions. Simulations and experiments have shown that the approach is sensitive to changes in the physical process conditions, such as changes in acid concentration (Orton et al. 2011, 2012). However, normal differences in the spent fuel can also lead to changes in the gamma spectrum. To further develop the MIP Monitor capability, we want to characterize the spent fuel sample via the gamma spectra in order to confirm inventory, develop fuel-specific baseline conditions for a nominal spectrum, and improve monitoring ability.

The current work aims to expand the MIP Monitor by characterizing SNF after initial dissolution at the front end of a reprocessing facility. In addition to confirming the declared fuel characteristics in reprocessing streams, this initial characterization can be used to increase the monitor's sensitivity to changes in later stages by using a fuel-specific set of expected nominal gamma spectra for subsequent monitoring and analysis.

\subsection{Data Simulation}

All the data used to develop and test the analysis methodology was generated using ORIGEN-ARP version 5.1.01 and ENDF/B-VI nuclear data (Bowman and Leal 2000). The development data consisted of three PWR and three BWR reactor designs, six initial enrichment values evenly distributed among typical commercial values, four cooling times, and nine discharge burn-up values. Spent fuel samples were simulated for each combination of reactor design, burn-up, initial enrichment, and cooling time shown in Table 2.1, resulting in a total of 1296 simulated fuel samples. The 200 nuclides with the highest activity in the ORIGEN-ARP output of each fuel sample were retained and used to simulate the gamma spectra of the fuels.

Table 2.1. Spent Fuel Characteristics Used for Data Simulation

\begin{tabular}{|c|c|c|c|c|}
\hline \multirow{2}{*}{$\frac{\text { Reactor Design }}{\text { CE14×14 (PWR) }}$} & \multicolumn{2}{|c|}{$\begin{array}{c}\text { Burn-up } \\
(\mathrm{MWd} / \mathbf{k g U})\end{array}$} & \multirow{2}{*}{$\begin{array}{c}\begin{array}{c}\text { Initial } \\
\text { Enrichment } \\
(\%)\end{array} \\
2.5\end{array}$} & \multirow{2}{*}{$\begin{array}{c}\begin{array}{c}\text { Cooling } \\
\text { Time } \\
\text { (years) }\end{array} \\
2\end{array}$} \\
\hline & 20 & 40 & & \\
\hline S14×14 (PWR) & 25 & 45 & 3.0 & 3 \\
\hline W17×17 (PWR) & 30 & 50 & 3.5 & 4 \\
\hline Atrium9×9 (BWR) & 34 & & 4.0 & 5 \\
\hline GE7×7 (BWR) & 35 & & 4.5 & \\
\hline SVEA64 (BWR) & 36 & & 5.0 & \\
\hline
\end{tabular}

The results of the ORIGEN-ARP analyses were then input to SYNTH, a program developed at PNNL to simulate the measured gamma spectra for a specified source. SYNTH allows the user to specify the physical characteristics of the source, as well as the constituent nuclides and their activities, the type of detector, the distance between the source and the detector, any intervening absorbers, and the electronics configuration, in order to fully define the gamma spectroscopy problem. For this research, in each 
simulation, the gamma source was treated as a point source $25 \mathrm{~cm}(\sim 10$ inches $)$ from the detector. Air was the only absorber between the source and detector. A coaxial high-purity germanium (HPGe) detector was used, with the detector and counting characteristics given in Table 2.2. The HPGe detector is simulated here as a "best-case scenario"; the excellent energy resolution of HPGe detectors will provide higher quality data for the multivariate analyses. However, in practice, HPGe detectors would likely not be employed because they must be continuously cooled (typically to $77 \mathrm{~K}$ ) to avoid thermally-induced leakage current. More likely, sodium iodide (NaI) or lanthanum bromide ( $\mathrm{LaBr}$ ) detectors would be deployed, but these do not produce the same quality of spectrum. Spectra collected with these detectors are expected to include similar pattern changes which the developed methodology could exploit for fuel characterization. Evaluating the efficacy of the developed algorithms and framework based on gamma spectra collected from these detectors is an area of future research.

Table 2.2. HPGe Detector and Electronics Parameters for SYNTH Gamma Spectra Simulations

\begin{tabular}{lll}
\hline \multirow{3}{*}{ End Cap } & Thickness & $0.5 \mathrm{~mm}$ \\
& Material & Aluminum \\
Dead Layer & Spacing & $0.666 \mathrm{~mm}$ \\
& Thickness & $0.666 \mathrm{~mm}$ \\
Sensor & Diameter & $6.66 \mathrm{~cm}$ \\
& Length & $6.66 \mathrm{~cm}$ \\
& Efficiency $132 \mathrm{keV}$ & $66.60 \%$ \\
\multirow{2}{*}{ Counting Parameters } & $1.666 \mathrm{keV}$ \\
& Resolution @1332 & $0 \mathrm{keV}$ \\
ADC & Zero & $1 \mathrm{keV} / \mathrm{ch}$ \\
Full Scale Energy & Quad & $0 \mathrm{keV} / \mathrm{ch}^{2}$ \\
\hline
\end{tabular}

\subsection{Data Analysis Methods}

Several multivariate analysis methods were investigated for application in this study, including methods for classification and estimation. The analysis methods ultimately employed in this research are described in the following sections.

Preprocessing input data before performing multivariate analysis can simplify calculations by removing systematic deviations that do not provide information about the process or system. Data preprocessing typically is performed within the variables, as opposed to within the observations. In the analyses presented here, the data are mean-centered (i.e., the mean of each variable in the training data is subtracted from each observation of that variable to give a post-processed variable mean of zero) to remove the large, but uninteresting, deviations from zero. Additionally, data are scaled to unit-variance within the variables to remove the relative effects of including input variables which have different units or are measured on different scales. For the analyses in this research, the inputs are all channels of a gamma spectrum, so they are fundamentally measured on the same scale; however, the range of responses in different channels can be very different, which causes the modeling methods to effectively assign larger "importance" to channels with larger variation. Variance scaling maintains relative differences in magnitude across observations of the same variable, but it can obscure differences in magnitude across 
variables of the same observation. In addition to these variable-based preprocessing methods, data observations can be scaled to reduce the effect of intensity. In gamma spectra analysis, this would be important if spectra are counted for different amounts of time. In this research, the counting time is kept constant, and so observation intensity is maintained because it is expected to contain important information for the spent fuel characterization. Other data preprocessing methods include noise reduction and maximizing the signal-to-noise ratio (SNR); because these data are simulated, it is assumed the spectra are noise free. In a deployed system, noise analysis and reduction may be more important.

The analysis models ultimately employed in characterizing spent fuel samples are all based on partial least squares regression. The following sections introduce partial least squares regression and two variations, partial least squares discriminant analysis for classification and locally weighted partial least squares for estimation.

\subsubsection{Partial Least Squares Regression}

Partial least squares regression (PLS) was originally developed in the field of econometrics, but has found more ubiquitous application in chemometrics. Practically, its application is not limited to chemical analyses; PLS is a linear regression model that capitalizes on the linear dependence, or co-linearity, of input variables, a feature of most data that makes it unsuited for traditional linear regression. A fully motivated derivation of PLS is given in Geladi and Kowalski (1986); an abbreviated derivation is given here.

PLS combines the attractive features of principal component analysis (PCA) with multiple linear regression. PCA is a method for identifying the underlying independent components in a set of correlated variables (Hotelling 1933a, b). The first principal component (PC) lies in the direction of greatest variance of the original data set. Subsequent PCs are orthogonal and lie in the direction of the greatest remaining variation. Each subsequent $\mathrm{PC}$ contains less information (variance) from the original data than the PCs before it. Typically, small PCs contain primarily noise and are excluded from the PC space, so that PCA can also be used as a dimension reduction technique.

PCA decomposes input variables into a set of independent components that explain the variance in the input variables. PLS extends PCA by identifying components (here called latent variables) that explain both the variance in the inputs and the covariance between the input and output variables. That is, the latent variables (LVs) are preferentially chosen to maximize their predictive potential for the dependent variables.

There are several iterative algorithms for defining the PLS model. The algorithm shown below is based on the one given in Hoffman et al. (2009). PLS training begins by identifying a direction, $\boldsymbol{u}_{\boldsymbol{l}}$, in the input space that highly correlates with the output. The input variables are projected on this direction to compute the corresponding regression coefficient, $\beta_{l}$. Further directions in the input space and regression coefficients are identified by deflation. Here, $X$ is the training input data, and $y$ is the training output data. In this implementation, the model predicts only one dependent variable; the algorithm can be expanded to include multiple dependent variables by iterating through the full training for each output variable. In effect, a separate PLS model is developed for each output, because the latent variables which best explain one output may not be those best suited for a different output. Because the rank of $y$ remains unchanged through PLS model development, the maximum number of LVs that can be identified is equal to the rank of $X$. Typically, however, the number of LVs is less than the rank of $X$ because, as in PCA, the later LVs 
likely contain primarily noise. The algorithm shown selects some preselected number of LVs, $k$. Another approach is to select LVs until the residual (the unexplained information in $y$, given by $y_{i+1}$ ) is smaller than some specified threshold.

Algorithm A: Partial Least Squares Regression Training and Prediction, from Hoffman et al. (2009)

\begin{tabular}{ll}
\hline Training: & Prediction: \\
\hline 1: $X_{1}=X$ & 1: $x_{1}=x$ \\
2: $y_{1}=y$ & 2: $y_{1}=0$ \\
3: for $i=1$ to $k$ do & 3: for $i=1$ to $k$ do \\
4: $\quad \boldsymbol{u}_{\boldsymbol{i}}=X_{i}^{T} y_{i}$ & $4: \quad \boldsymbol{s}_{\boldsymbol{i}}=x_{i}^{T} \boldsymbol{u}_{\boldsymbol{i}}$ \\
5: $\quad \boldsymbol{s}_{\boldsymbol{i}}=X_{i} \boldsymbol{u}_{\boldsymbol{i}}$ & $5: \quad y_{i+1}=y_{i}+\beta_{i} \boldsymbol{s}_{\boldsymbol{i}}$ \\
6: $\quad \beta_{i}=\boldsymbol{s}_{i}^{T} y_{i} /\left(\boldsymbol{s}_{\boldsymbol{i}}^{T} \boldsymbol{s}_{\boldsymbol{i}}\right)$ & $6: \quad x_{i+1}=x_{i}-\boldsymbol{s}_{\boldsymbol{i}} \boldsymbol{p}_{\boldsymbol{i}}$ \\
7: $\quad y_{i+1}=y_{i}-\beta_{i} \boldsymbol{s}_{\boldsymbol{i}}$ & $7:$ end for \\
8: $\quad \boldsymbol{p}_{\boldsymbol{i}}=X_{i}^{T} \boldsymbol{s}_{\boldsymbol{i}} /\left(\boldsymbol{s}_{\boldsymbol{i}}^{T} \boldsymbol{s}_{\boldsymbol{i}}\right)$ & $8: \hat{y}=y_{k+1}$ \\
9: $\quad X_{i+1}=X_{i}-\boldsymbol{s}_{\boldsymbol{i}} \boldsymbol{p}_{\boldsymbol{i}}^{T}$ & \\
10: end for & \\
\hline
\end{tabular}

\subsubsection{PLS Discriminant Analysis}

The PLS algorithm described above can be adapted for classification purposes (Barker and Rayens 2003); this adaptation is commonly referred to as PLS discriminate analysis (PLSDA). In PLSDA, the output training block is a matrix of binary dummy variables indicating whether or not an observation belongs to a specific class:

$$
y_{i j}=\left\{\begin{array}{cc}
1 & \text { if obs } i \in \text { class } \mathrm{j} \\
0 & \text { otherwise }
\end{array}\right.
$$

This encoding will help the PLS training algorithm identify between-group variance that is useful to discriminate between classes. In the prediction step, the membership of a new observation in each class is estimated. By design, this prediction is continuous; a threshold is applied to convert it to a binary measure. If the predicted membership is above the threshold, then the observation belongs to that class; below the threshold, and it does not.

This formulation introduces the possibility that an observation may be classified as belonging to multiple classes, or to no class. To avoid this, a strict maximum can be applied to the predicted memberships so that the observation is assigned only to the class to which its predicted membership is highest. The appropriate classification rule depends on the system under study and the goals of the classification. In this research, the threshold method is used. 


\subsubsection{Locally Weighted PLS}

Most nonlinear surfaces can be considered approximately linear in some small neighborhood about a point. Locally weighted methods are often used to apply linear methods to these nonlinear problems by considering only the data within that linear neighborhood when training the models. PLS, as described above, is inherently linear. PLS can accommodate small nonlinearities, but the nonlinear information will be lost when transforming from the original input space to LVs. When data contain significant nonlinearities, PLS tends to fall apart. Locally weighted PLS (LWPLS) can preserve the globally nonlinear information by creating locally linear models as new observations are made. LWPLS has been applied to a number of problems (Schaal et al. 2000; Kim et al. 2011; Liu et al. 2012).

The LWPLS algorithm is similar to the PLS algorithm presented above. The primary difference is the inclusion of a weighting matrix when evaluating the principal directions, $\boldsymbol{u}_{\boldsymbol{i}}$, the regression coefficients, $\beta_{i}$, and the projection of $X$ on $\boldsymbol{u}_{i}, \boldsymbol{p}_{i}$. The locally weighted calculations are given by:

$$
\begin{aligned}
& \boldsymbol{u}_{\boldsymbol{i}}=X^{T} W y_{i}, \\
& \beta_{i}=\boldsymbol{s}_{\boldsymbol{i}}^{T} W y_{i} /\left(\boldsymbol{s}_{\boldsymbol{i}}^{T} W \boldsymbol{s}_{\boldsymbol{i}}\right), \text { and } \\
& \boldsymbol{p}_{\boldsymbol{i}}=X_{i}^{T} W \boldsymbol{s}_{\boldsymbol{i}} /\left(\boldsymbol{s}_{\boldsymbol{i}}^{T} W \boldsymbol{s}_{\boldsymbol{i}}\right),
\end{aligned}
$$

where $W$ is a diagonal matrix containing the weight for each exemplar input/output pair in $X$ and $y$. The weights can be defined in any way that indicates the similarity of a new observation to the historic (exemplar) data. A variety of approaches have been employed, including moving windows for timeseries data (Liu et al. 2012) and Gaussian kernel weighting based on the similarity of the inputs (Schaal et al. 2000; Kim et al. 2011).

The current research takes a similar approach to kernel weighting; however, the weights are calculated by the similarity of the fuel characteristics which have already been estimated for the new observation (i.e., reactor type, burn up, initial enrichment, and/or cooling time) to the actual, known values of these characteristics for the exemplar data. The weighting scheme used here does not consider how similar a new spectrum is to the exemplar spectra. The weight of the $i^{\text {th }}$ exemplar observation for a new prediction is given by the product of the available estimated characteristic similarities:

$$
w_{i}=\prod_{k} w\left(\hat{c}_{k}, c_{i, k}\right)
$$

where $k$ is the number of characteristics that have been estimated, $\hat{c}_{k}$ is the estimated value of the $k^{\text {th }}$ characteristic for the new observation and $c_{i, k}$ is the known value of the $k^{\text {th }}$ characteristic for the $i^{\text {th }}$ exemplar. The method of calculating the characteristic similarity is slightly different for classifications (e.g., reactor type) and estimations (e.g., burn up, initial enrichment, or cooling time). The similarity associated with reactor type is binary:

$$
w\left(\hat{c}_{R x}, c_{i, R x}\right)= \begin{cases}1 & \hat{c}_{R x}=c_{i, R x} \\ 0 & \hat{c}_{R x} \neq c_{i, R x}\end{cases}
$$


such that the resulting $w_{i}$ will necessarily be zero if the exemplar observation is from a different reactor type than estimated for the new observation. The similarity of the continuous characteristics is evaluated with the Gaussian kernel. First, the distance between the estimated characteristic of the new observation and the known value of the exemplar is calculated. Most commonly, the Euclidean distance is used, which simplifies to the $\mathrm{L}_{1}$ norm when only one dimension (here, fuel characteristic) is considered at a time:

The distance is then converted to a similarity measure through the Gaussian kernel:

$$
d_{k}=\sqrt{\left(\hat{c}_{k}-c_{i, k}\right)^{2}}=\left|\hat{c}_{k}-c_{i, k}\right| .
$$

$$
w\left(\hat{c}_{k}, c_{i, k}\right)=\exp \left(-\frac{d_{k}^{2}}{h_{k}^{2}}\right)
$$

where $h_{k}$ is the kernel bandwidth, which effectively controls how close the estimated characteristic and the known exemplar characteristic must be to be considered similar. The kernel bandwidth should be chosen for each characteristic of interest, based on the range of values of the characteristic and the additional predictive power gained by including that information in the model. As $h_{k}$ approaches zero, Eq. (6) approaches the binary weighting in Eq. (4); conversely, as $h_{k}$ approaches infinity, the weighting function in Eq. (6) approaches a constant value of $w$ equal to one.

\subsubsection{Input Selection}

Input selection is a common first step to model development. In general, including inputs which are not useful to the model increases the noise in the model predictions, while withholding important inputs will introduce a prediction bias. Therefore, it is important to ensure that the correct set of inputs is used. Identifying the correct inputs is relatively straightforward when the inputs are measurements of disparate parameters (such as flow, temperature, pressure, etc.). In this application, the input space consists of the channels of gamma spectra. Here, it is difficult and likely unwise to consider the channels individually. A specific peak will span several channels, and slight changes in the peak shape may occur as the result of the random nature of radiation. For these reasons, it makes sense to choose contiguous groups of channels instead of choosing channels individually.

Several methods exist for performing input selection specifically for PLS models (Alsberg et al. 1998; Höskuldsson 2001; Brás et al. 2008; Andersen and Bro 2010; Kim et al. 2011). The interval-PLS (iPLS) method was used in this research (Norgaard et al. 2000) [PLS Toolbox help (Wiki 2011)]. iPLS is a brute force search method that identifies a subset of inputs that give superior predictive performance compared to the full input space. In this input selection approach, each interval of a specified size is evaluated to determine which single interval results in the best model performance during cross validation. Once the single most useful interval has been identified, this interval is used in all the models evaluated during the second pass. Each remaining interval is combined with the identified "best" interval to develop a model and evaluate its performance. This exhaustive search continues, where the set of previously identified intervals is combined with each of the remaining intervals to identify the next most useful one. The interval selection ends when the cross validation error no longer improves with the addition of any 
interval. For this research, an interval size of five channels is used for all input selection. This interval width was selected based on the peak widths seen in the simulated gamma spectra.

\subsection{Implementation}

The analysis method has been implemented in MATLAB R2011b in both a command line and GUI implementation shown in Figure 2.1. Where a static (i.e., not locally-weighted) model is used for classification or prediction, the model has been trained and saved; the appropriate static models are loaded into the analysis where appropriate. Although the full training set is available for the locally-weighted PLS models, loading pre-trained PLS and PLSDA models saves significant runtime by not requiring that these be retrained with each new observation.

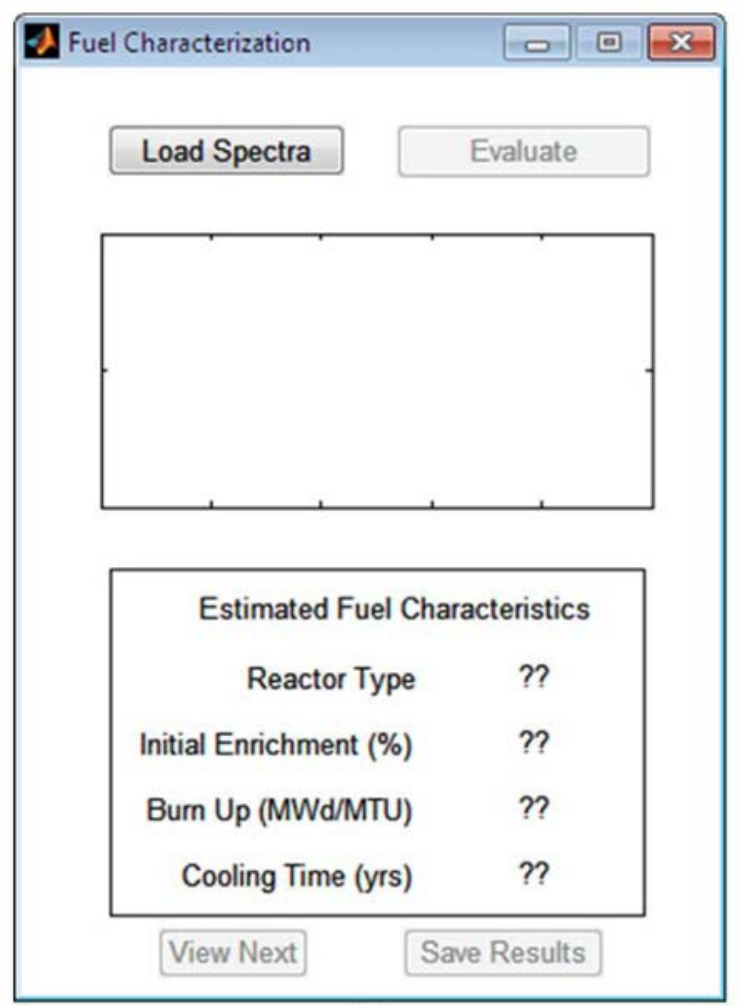

(a)

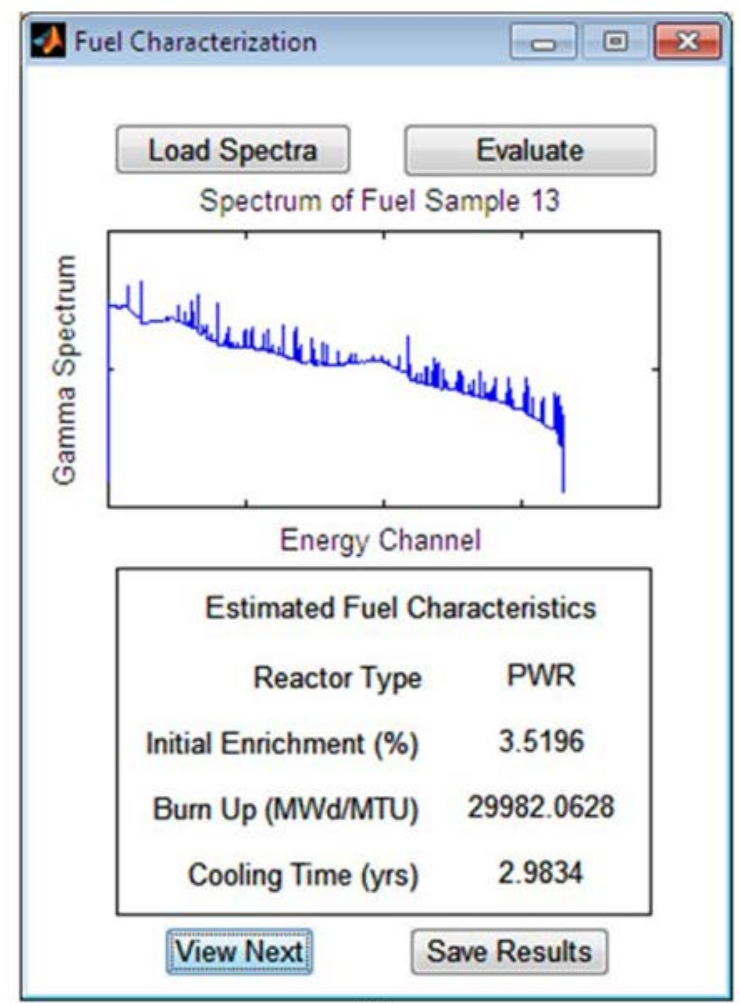

(b)

Figure 2.1. Fuel Characterization GUI (a) Before Spectra are Loaded and (b) After Spectra are Evaluated. Additional shots of the GUI are included in Appendix B.

The MIP Monitor works in real-time (or NRT) to monitor reprocessing streams. Generally, the monitor is expected to use 30-second spectra (or longer); therefore, the multivariate analysis methods employed should complete predictions in under 30 seconds. This implementation was evaluated on a 3.40-GHz Intel Pentium 4 processor. Execution speed for a single sample was evaluated by averaging the elapsed time for 324 validation samples. The MATLAB implementation of Algorithm B, described in the next section, averaged 1.85 seconds per evaluation. 



\subsection{Results}

This section summarizes the results of the data simulation and multivariate analysis employed in this research.

\subsection{Simulation Results}

The top 200 nuclides ranked by concentration at discharge were output from ORIGEN-ARP for each fuel sample and the resulting nuclide vectors were used as input observations for the analysis algorithms. In total, 218 distinct nuclides were selected from the ORIGEN-ARP output. The included nuclides are given in Appendix A. The activities of all 218 nuclides were used to generate simulated gamma spectra with SYNTH. The 1296 resulting spectra are plotted in Figure 3.1. These spectra are used in the multivariate analyses to characterize the spent fuel properties.

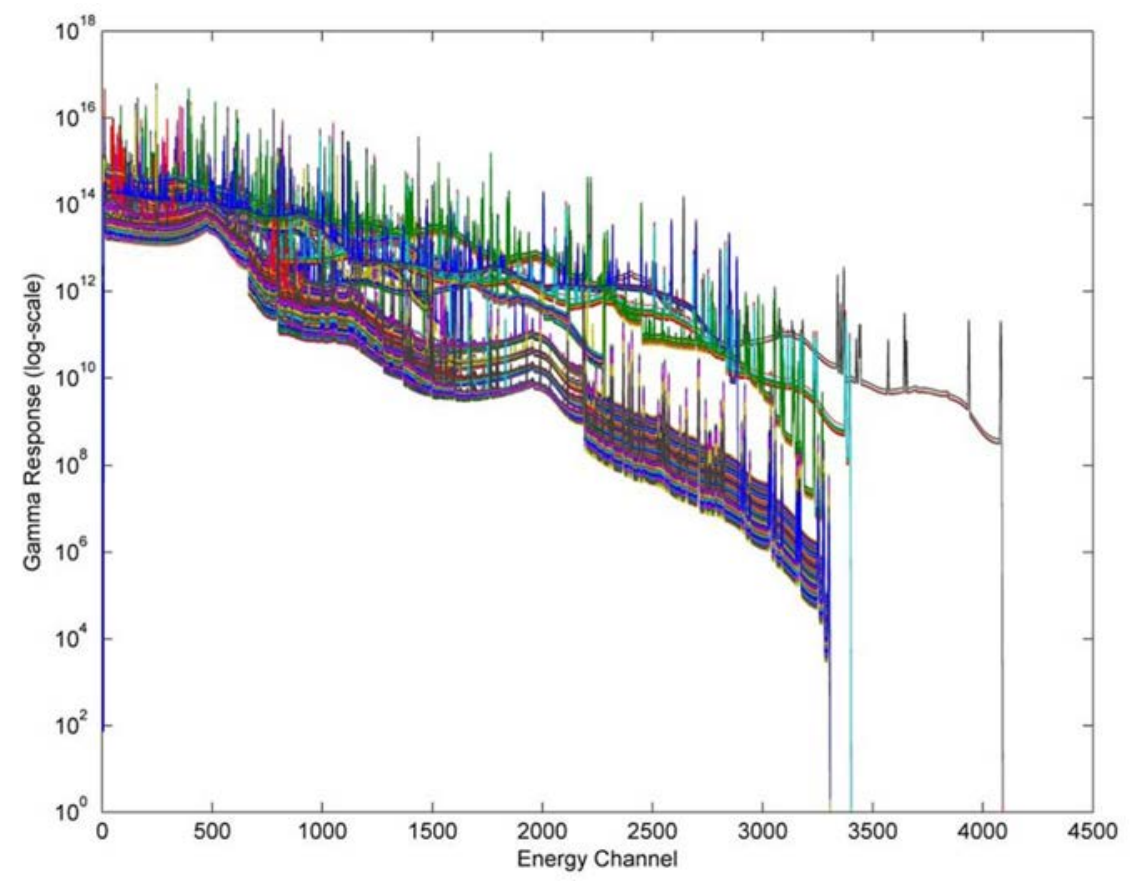

Figure 3.1. Simulated Gamma Spectra for 1296 Example Spent Fuel Samples

The fidelity of the simulation results depends on the fidelity of each of the codes used. A number of studies that benchmark ORIGEN-ARP/Origen-S and the nuclide inventories these codes generate have been performed (Gauld and Litwin 1995; Leal et al. 1999; Gauld and Murphy 2004). These benchmark studies report the error in prediction made by ORIGEN-ARP/Origen-S over the measured values in SNF for some of the nuclides used in this research. The relevant results of are summarized in Table 3.1.

These results include only benchmarking exercises which evaluate spent fuel samples within the range of characteristics considered. Of the 14 nuclides benchmarked, 9 have reported error less than $10 \%$. Conversely, two nuclides, ${ }^{126} \mathrm{Sn}$ and ${ }^{79} \mathrm{Se}$, have shown significant prediction error in benchmarking tests. These errors are typically attributed to poorly characterized cross-section data. Despite these and 
potentially other large errors, we expect that the overall trends in variation between samples give a realistic view of the kinds of variations expected in practice, because the errors in nuclear data are present in every simulation. The multivariate analysis methods used in the MIP Monitor capitalize on these variations between samples to characterize the fuel sample, not the overall magnitude of the variables. The models developed in this research are not field-deployable, but they suggest that similar analyses can be performed on real-world data.

Table 3.1. Results of ORIGEN-ARP Nuclide Benchmarking

\begin{tabular}{crc}
\hline Nuclide & Error [\%] & Reference \\
\hline${ }^{238} \mathrm{Pu}$ & 10.98 & Gauld and Litwin (1995), Leal et al. (1999), Gauld and Murphy (2004) \\
${ }^{239} \mathrm{Pu}$ & 4.91 & Gauld and Litwin (1995), Leal et al. (1999), Gauld and Murphy (2004) \\
${ }^{240} \mathrm{Pu}$ & 4.08 & Gauld and Litwin (1995), Leal et al. (1999), Gauld and Murphy (2004) \\
${ }^{241} \mathrm{Pu}$ & 5.41 & Gauld and Litwin (1995), Leal et al. (1999), Gauld and Murphy \\
& & (Gauld and Litwin 1995; Leal et al. 1999; 2004) \\
${ }^{99} \mathrm{Tc}$ & 8.40 & Gauld and Litwin (1995), Leal et al. (1999) \\
${ }^{90} \mathrm{Sr}$ & 9.00 & Gauld and Litwin (1995) \\
${ }^{137} \mathrm{Cs}$ & 1.20 & Gauld and Litwin (1995), Leal et al. (1999), Gauld and Murphy (2004) \\
${ }^{154} \mathrm{Eu}$ & 1.00 & Gauld and Murphy (2004) \\
${ }^{237} \mathrm{~Np}$ & 7.58 & Gauld and Litwin (1995), Leal et al. (1999) \\
${ }^{151} \mathrm{Sm}$ & 17.00 & Gauld and Litwin (1995), Gauld and Murphy (2004) \\
${ }^{126} \mathrm{Sn}$ & 202.00 & Gauld and Litwin (1995) \\
${ }^{79} \mathrm{Se}$ & 980.00 & Gauld and Litwin (1995) \\
${ }^{242 \mathrm{~m}} \mathrm{Am}$ & 2.00 & Gauld and Murphy (2004) \\
${ }^{243} \mathrm{Cm}$ & 22.00 & Gauld and Murphy (2004) \\
\hline
\end{tabular}

The simulated data were divided into three data sets for model development and testing. The training set includes 722 observations, which cover the range of simulated values in each channel, including the minimum and maximum values of each channel, and the range of defined characteristics (reactor type, initial enrichment, burn up, and cooling time). The data contained in the training set are used to train the PLS-based models. The test data set contains 324 observations; this data is used to optimize the trained models for the number of latent variables and the weighting bandwidths (in LWPLS models). Finally, the validation set contains 324 observations, which are used to evaluate the performance of the final models. The model error metrics presented in the following section are all evaluated on the validation data set.

\subsection{Fuel Characterization}

One of the main focuses of this research was to determine the appropriate order to characterize the four fuel features of interest - reactor type, initial enrichment, burn up, and cooling time. When these characteristics are evaluated sequentially, the features that have already been predicted can be used along with the gamma spectra to improve the predictive performance. Several fuel characterization orders were considered, in addition to performing all predictions in parallel (i.e., using only the gamma spectra to characterize all four features of the spent fuel). 
In all the algorithms tested, first classifying the sample's reactor type seemed to be the single best way to improve model predictions. A PLSDA model was trained that perfectly classifies the simulated fuel samples into PWR and BWR fuel types. This PLSDA model uses five intervals in the spectrum to determine which class a sample belongs to. The five selected channel ranges are illustrated in Figure 3.2 by the red highlighted portions of the spectra. SYNTH provides a table of some of the key contributors to peaks in the simulated gamma spectrum. This report does not reach the full channel length of the gamma spectrum, but three of the five selected ranges are included. The top contributors to these ranges are shown in Table 3.2. The same PLSDA model is used in each algorithm for fuel classification.

In addition to the parallel prediction, the results of two algorithms are presented here. Algorithm A classifies the reactor type of the sample, then uses reactor type-specific static PLS models to predict each of the three remaining characteristics. Algorithm B first classifies the sample, then uses a reactor typespecific static model (PLS) to predict burn up, and then uses the predicted burn up to estimate cooling time with LWPLS, and finally predicts initial enrichment using both cooling time and burn up using LWPLS. Flow charts for Algorithm A and Algorithm B are shown in Figure 3.3 and Figure 3.4, respectively. In all of the models, iPLS was used to select the most appropriate subset of features for each model. iPLS was used to select inputs for the parallel prediction models using all of the training data. For the prediction models in Algorithms A and B, the training data was divided by the two reactor types, and appropriate inputs for each model were selected for each reactor type. In total, six sets of features were selected for prediction models, a separate set for initial enrichment, burn up, and cooling time for both PWRs and BWRs. The same inputs were used for models in both Algorithm A and B.

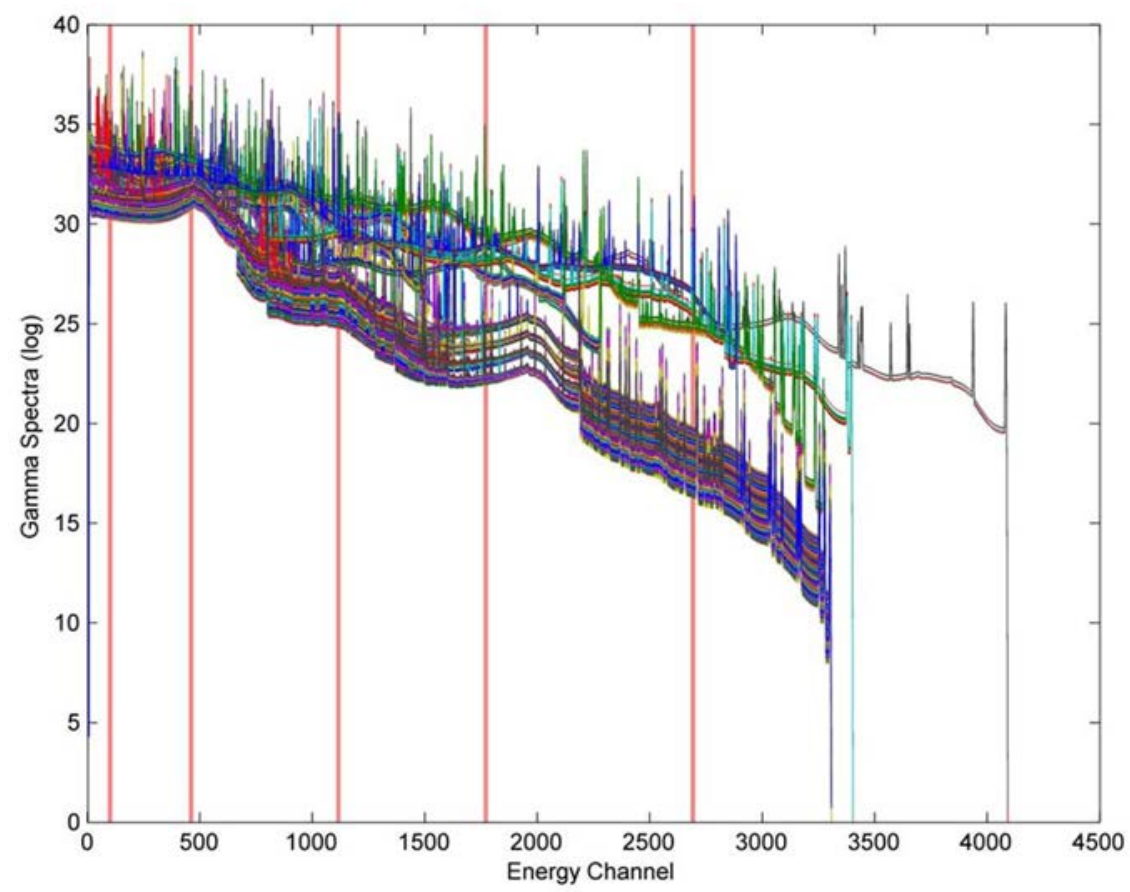

Figure 3.2. Selected Channels for Reactor Type Classification with PLSDA 
Table 3.2. Key Gamma Contributors for Reactor Type Classification

\begin{tabular}{cc}
\hline Channel Range & Key Contributors \\
\hline & Bi-214 \\
& Eu-155 \\
& Ce-144 \\
& Np-239 \\
& Cm-243 \\
Te-125m \\
Sb-110 \\
& Pu-241 \\
& Bi-214 \\
& $\mathrm{Sb}-125$ \\
& $\mathrm{Rh}-106$ \\
$451: 470$ & $\mathrm{Eu}-154$ \\
& $\mathrm{Bi}-214$ \\
& $\mathrm{Rh}-106$ \\
& $\mathrm{Eu}-154$ \\
& $\mathrm{Eu}-152$ \\
\hline
\end{tabular}

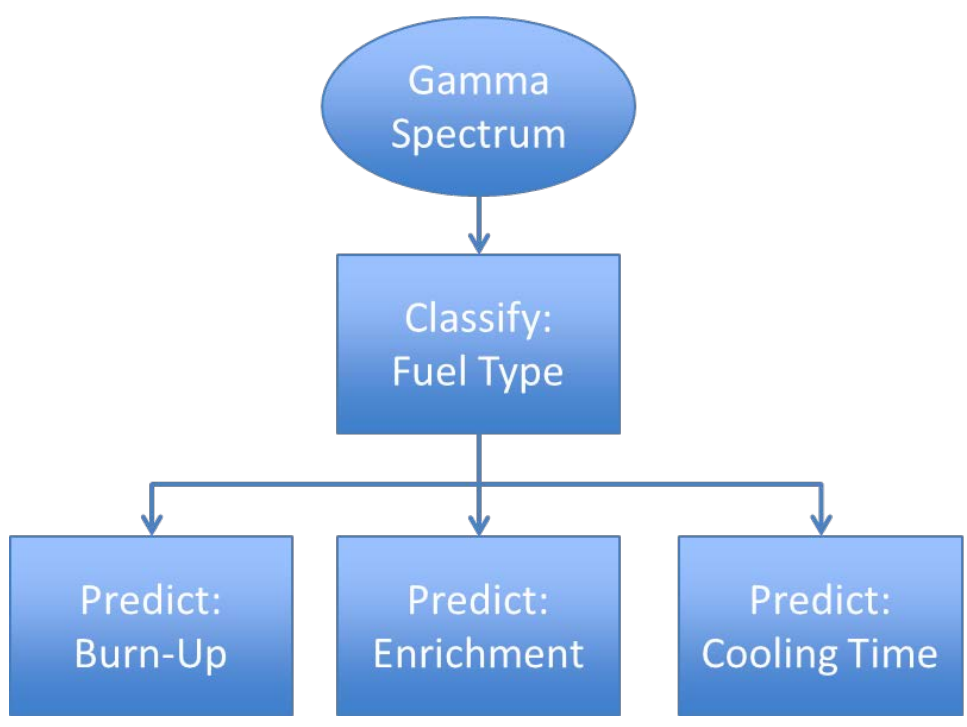

Figure 3.3. Flow Diagram for Algorithm A

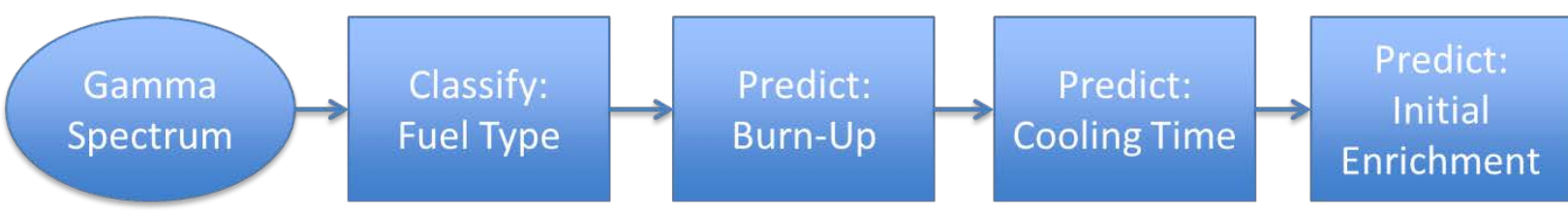

Figure 3.4. Flow Diagram for Algorithm B 
The results for all three algorithms are summarized in Table 3.3. Results are given as the root mean squared percent error (RMSPE), which is calculated as:

$$
\operatorname{RMSPE}=\sqrt{\frac{\sum(\hat{y}-y)^{2}}{n}} / E[p] \times 100 \%,
$$

where $\hat{y}$ is the prediction of some feature $y$ for $n$ observations, and $E[y]$ is the expected value (mean) of $y$. In all cases, the reactor type can be perfectly classified using the PLSDA model described above.

Algorithm A shows significant improvement over parallel characterization, with improvements of a factor of two to four for prediction of initial enrichment, burn up, and cooling time. Algorithm B shows further improvements over Algorithm A for prediction of initial enrichment and cooling time. The same reactorspecific PLS models are used to predict burn up in both Algorithm A and B, giving a prediction RMSPE on the validation set of approximately $0.1 \%$. The results of the multivariate analyses presented here may be unrealistically accurate, because the data do not contain noise or random perturbations. However, the results suggest that fuel characterization can be effectively performed on dissolved spent fuel samples using gamma spectroscopy.

Table 3.3. Fuel Characterization RMSPE for Parallel Prediction, Algorithm A, and Algorithm B

\begin{tabular}{lccccccccc}
\hline & \multicolumn{3}{c}{ Parallel Characterization } & \multicolumn{3}{c}{ Algorithm A } & \multicolumn{3}{c}{ Algorithm B } \\
\cline { 2 - 10 } & Overall & PWR & BWR & Overall & PWR & BWR & Overall & PWR & BWR \\
\hline Reactor Type & 0.00 & 0.00 & 0.00 & 0.00 & 0.00 & 0.00 & 0.00 & 0.00 & 0.00 \\
Initial Enrichment & 10.94 & 10.54 & 11.32 & 2.71 & 3.13 & 2.23 & 1.78 & 1.69 & 1.87 \\
Burn Up & 0.36 & 0.42 & 0.29 & 0.11 & 0.09 & 0.13 & 0.11 & 0.09 & 0.13 \\
Cooling Time & 6.62 & 8.65 & 3.81 & 3.39 & 2.96 & 3.79 & 2.13 & 1.26 & 2.80 \\
\hline
\end{tabular}





\subsection{Conclusions}

The research presented in this report extends the previously developed MIP Monitor by developing a methodology for characterizing dissolved spent fuel samples according to reactor type (PWR or BWR), initial enrichment, burn up, and cooling time based on gamma spectra. Two main objectives are met by characterizing and classifying the spent fuel at initial dissolution: (1) verifying that the fuel is consistent with written inventories and (2) allowing for fuel-specific multivariate models to monitor conditions later in the process. Using fuel-specific multivariate models will improve the sensitivity of the MIP Monitor to small perturbations and anomalies in the process. This initial study used simulated data from ORIGENARP and SYNTH to develop models and algorithms for this fuel characterization. Despite the limitations of using simulated data instead of actual data, the performance of the models developed in this research suggest that similar analyses can be performed on actual gamma spectra collected from dissolved fuel. The concepts and framework developed are generically applicable, though the specific models used will need to be developed and trained on actual, experimental, or operational data.

Several important lessons in developing a fuel characterization algorithm have been learned in this research. First, the estimations of initial enrichment, burn up, and cooling time were all improved by first classifying the fuel as from a PWR or BWR. Predictions of cooling time and initial enrichment could be further improved by including the predicted burn up and both burn up and cooling time, respectively. Input selection was used for each model to improve model performance and stability. The improved model performance seen after selecting a subset of intervals in the full input space suggests that the multivariate models are targeting key nuclides through specific regions in the spectrum. It may be interesting to identify the nuclides which contribute to the selected peaks for each of the prediction models as was done for the reactor type classifier; this analysis is left to future work.

The aim of this research was to develop an algorithm for characterizing SNF samples based on simulated gamma spectra. These spectra were evaluated with PLS-based multivariate analysis methods to characterize the fuel according to reactor type (PWR or BWR), enrichment, burn up, and cooling time. Characterizing some of the features in series by using previously estimated features in the prediction greatly improves the performance. By first classifying the spent fuel reactor type and then using typespecific models, the prediction error for enrichment, burn up, and cooling time improved by a factor of two to four. For some features, the prediction was further improved by including additional information, such as including the predicted burn up in the estimation of cooling time. The optimal prediction flow was determined based on the simulated data to be a series analyses of reactor type, burn up, cooling time, and initial enrichment, where each prediction in the series uses the estimations of all prior predictions. A PLS discriminate analysis model was developed which perfectly classified SNF reactor type. Burn up was predicted within $0.1 \%$ root mean squared percent error and both cooling time and initial enrichment within approximately $2 \%$ RMSPE. 



\subsection{Future Work}

The research presented here suggests that dissolved spent fuel samples can be characterized by reactor type, initial enrichment, burn up, and cooling time using the gamma spectrum. However, several areas of additional research remain before the fuel characterization algorithm can be deployed. Some key areas of needed research are outlined here.

The models and algorithm developed in this research are based on simulated, noise-free data. While this research suggests that application to real-world data will be successful, the models are not directly applicable to operational data. There are two paths to develop models and an algorithm for deployment. The first path continues to focus on simulated data, but will involve significant work to evaluate and improve the efficacy of these simulations. The fidelity of ORIGEN-ARP simulations should be evaluated for the range of fuel characteristics employed and the 218 nuclides enumerated. This work has already begun, as discussed in the results section; however, the vast majority of the nuclides have not been benchmarked for fuel samples similar to those used here. The gamma spectra resulting from SYNTH also need to be evaluated and benchmarked for fidelity. Additionally, neither simulation accounts for the noise inherent in the system, both process noise and random noise. The ORIGEN-ARP simulation is deterministic; however, there will be variations in the nuclide concentrations due to variations in manufacturing, location in the reactor core and in the fuel pin, differences due to the random nature of radioactive decay, etc. The gamma spectra also suffer from counting noise due to the random nature of radiation. The research presented here did not consider any of these noise terms in simulation or multivariate analysis. The second path to address this concern is to collect and evaluate real-world data, either in lab-scale experiments or from actual operations. Using real-world data will alleviate questions about the fidelity of data and the analysis methods, but it may be difficult to collect a sufficient database of gamma spectra from different fuel samples to develop these models. A proposal to instrument the fuel processing facility at the U.S. Department of Energy Savannah River Site (H-Canyon) is currently being pursued to support this data collection.

Because of the noise sources mentioned above and the uncertainty inherent in multivariate analysis, the fuel characterization should include a measure of uncertainty or confidence; for example, a confidence level of the classification of reactor type or confidence intervals for the initial enrichment, burn up, and cooling time. This will give a more realistic view of the fuel characterization results. These uncertainties will propagate through later monitoring tasks, such as anomaly detection. Understanding the prediction uncertainties and their effects on future analysis is particularly important. 



\subsection{References}

Alsberg BK, DB Kell and R Goodacre. 1998. "Variable Selection in Discriminant Partial Least-Squares Analysis." Analytical Chemistry 70(19):4126-4133.

Andersen CM and R Bro. 2010. "Variable Selection in Regression-A Tutorial." Journal of Chemometrics 24(11-12):728-737.

Barker M and W Rayens. 2003. "Partial Least Squares for Discrimination." Journal of Chemometrics 17(3):166-173.

Bowman SM and LC Leal. 2000. ORIGEN-ARP: Automatic Rapid Process for Spent Fuel Depletion, Decay, and Source Term Analysis. NUREG/CR-0200, U.S. Nuclear Regulatory Commission, Washington, D.C.

Brás LP, M Lopes, AP Ferreira and JC Menezes. 2008. "A Bootstrap-based Strategy for Spectral Interval Selection in PLS Regression." Journal of Chemometrics 22(11-12):695-700.

Gauld I and K Litwin. 1995. Verification and Validation of the ORIGEN-S Code and Nuclear Data Libraries. Report No. COG-I-95-150, Atomic Energy of Canada Limited, Ontario, Canada.

Gauld IC and BD Murphy. 2004. Updates to the Origen-S Data Libraries Using ENDF/B-VI, FENDL2.0, and EAF-99 Data. ORNL/TM-2003/118, Oak Ridge National Laboratory, Oak Ridge, Tennessee.

Geladi P and BR Kowalski. 1986. "Partial Least-Squares Regression: A Tutorial." Analytica Chimica Acta 185(0):1-17.

Hoffmann H, S Schaal and S Vijayakumar. 2009. "Local Dimensionality Reduction for Non-Parametric Regression." Neural Processing Letters 29(2):109-131.

Höskuldsson A. 2001. "Variable and Subset Selection in PLS Regression." Chemometrics and Intelligent Laboratory Systems 55(1-2):23-38.

Hotelling H. 1933a. "Analysis of a Complex of Statistical Variables into Principal Components." Journal of Educational Psychology 24(6):417-441.

Hotelling H. 1933b. "Analysis of a Complex of Statistical Variables into Principal Components." Journal of Educational Psychology 24(7):498-520.

Kim S, M Kano, H Nakagawa and S Hasebe. 2011. "Estimation of Active Pharmaceutical Ingredients Content Using Locally Weighted Partial Least Squares and Statistical Wavelength Selection." International Journal of Pharmaceutics 421(2):269-274.

Leal LC, OW Hermann, SM Bowman and CV Parks. 1999. "Automatic Rapid Process for the Generation of Problem-Dependent SAS2H/ORIGEN-S Cross-Section Libraries." Nuclear Technology 127(1):1-23.

Liu J, D-S Chen and M-W Lee. 2012. "Adaptive Soft Sensors Using Local Partial Least Squares with Moving Window Approach." Asia-Pacific Journal of Chemical Engineering 7:S134-S144. 
Norgaard L, A Saudland, J Wagner, JP Nielsen, L Munck and SB Engelsen. 2000. "Interval Partial Least-Squares Regression (iPLS): A Comparative Chemometric Study with an Example from NearInfrared Spectroscopy." Applied Spectroscopy 54(3):413-419.

Orton CR, CG Fraga, RN Christensen and JM Schwantes. 2011. "Proof of Concept Simulations of the Multi-Isotope Process Monitor: An Online, Nondestructive, Near-Real-Time Safeguards Monitor for Nuclear Fuel Reprocessing Facilities." Nuclear Instruments and Methods in Physics Research Section A: Accelerators, Spectrometers, Detectors and Associated Equipment 629(1):209-219.

Orton CR, CG Fraga, RN Christensen and JM Schwantes. 2012. "Proof of Concept Experiments of the Multi-Isotope Process Monitor: An Online, Nondestructive, Near Real-Time Monitor for Spent Nuclear Fuel Reprocessing Facilities." Nuclear Instruments and Methods in Physics Research Section A: Accelerators, Spectrometers, Detectors and Associated Equipment 672:38-45.

OTA. 1995. Nuclear Safeguards and the International Atomic Energy Agency. Report No. OTA-ISS615, Office of Technology Assessment (OTA), Washington, D.C.

Schaal S, CG Atkeson and S Vijayakumar. 2000. "Real-Time Robot Learning with Locally Weighted Statistical Learning." In Proceedings of IEEE International Conference on Robotics and Automation Proceedings (ICRA '00), pp. 288-293. April 24-28, 2000, San Francisco. The Institute of Electrical and Electronics Engineering, Inc. (IEEE), Piscataway, New Jersey.

Wiki. 2011. Interval PLS (IPLS) for Variable Selection. Wikipedia. Accessed July 24, 2012 Available at http://wiki.eigenvector.com/index.php?title=Interval_PLS_(IPLS)_for_Variable_Selection. 
Appendix A

Simulated Nuclides from ORIGIN-ARP 



\section{Appendix A}

\section{Simulated Nuclides from ORIGIN-ARP}

The following nuclides were simulated in ORIGEN-ARP for each example reactor and input to SYNTH to simulate the gamma spectrum of each dissolved fuel sample.

\begin{tabular}{|c|c|c|c|c|c|}
\hline Ac225 & Cf249 & In114 & Pm148 & Rn219 & Te127 \\
\hline Ac227 & Cf250 & In114m & Pm148m & Rn220 & $\mathrm{Te} 127 \mathrm{~m}$ \\
\hline Ag108 & Cf251 & In $115 \mathrm{~m}$ & Po210 & Rn222 & Te129 \\
\hline Ag108m & Cf252 & Kr81 & Po211 & Ru103 & Te129m \\
\hline Ag109m & Cf254 & Kr85 & Po212 & Ru106 & Th227 \\
\hline Ag110 & $\mathrm{Cm} 241$ & La137 & Po213 & $\mathrm{Sb} 124$ & Th228 \\
\hline Ag110m & $\mathrm{Cm} 242$ & La140 & Po214 & Sb125 & Th229 \\
\hline Ag111 & $\mathrm{Cm} 243$ & Mo93 & Po215 & $\mathrm{Sb} 126$ & Th230 \\
\hline Ag112 & $\mathrm{Cm} 244$ & Nb91 & Po216 & $\mathrm{Sb} 126 \mathrm{~m}$ & Th231 \\
\hline Am241 & $\mathrm{Cm} 245$ & Nb93m & Po218 & $\mathrm{Se} 75$ & Th234 \\
\hline Am242 & $\mathrm{Cm} 246$ & Nb94 & $\operatorname{Pr} 143$ & $\mathrm{Se} 79$ & $\mathrm{~T} 1207$ \\
\hline $\mathrm{Am} 242 \mathrm{~m}$ & $\mathrm{Cm} 247$ & $\mathrm{Nb95}$ & $\operatorname{Pr} 144$ & Sm145 & $\mathrm{T} 1208$ \\
\hline Am243 & $\mathrm{Cm} 248$ & $\mathrm{Nb95m}$ & $\operatorname{Pr} 144 m$ & Sm146 & T1209 \\
\hline Am 245 & Cs134 & Nb96 & $\operatorname{Pr} 145$ & Sm147 & Tm168 \\
\hline As 73 & Cs135 & Nd147 & $\mathrm{Pu} 236$ & Sm151 & $\operatorname{Tm} 170$ \\
\hline At217 & Cs136 & Np235 & $\mathrm{Pu} 237$ & $\mathrm{Sn} 117 \mathrm{~m}$ & $\operatorname{Tm} 171$ \\
\hline Ba133 & Cs137 & Np236 & $\mathrm{Pu} 238$ & $\mathrm{Sn} 119 \mathrm{~m}$ & $\mathrm{U} 232$ \\
\hline $\mathrm{Ba} 136 \mathrm{~m}$ & Cs138 & $\mathrm{Np} 237$ & Pu239 & Sn121 & $\mathrm{U} 233$ \\
\hline $\mathrm{Ba} 137 \mathrm{~m}$ & Dy159 & Np238 & $\mathrm{Pu} 240$ & $\mathrm{Sn} 121 \mathrm{~m}$ & $\mathrm{U} 234$ \\
\hline Ba140 & Dy166 & Np239 & $\mathrm{Pu} 241$ & Sn123 & $\mathrm{U} 235$ \\
\hline Be10 & Es254 & $\mathrm{Np} 240 \mathrm{~m}$ & $\mathrm{Pu} 242$ & $\mathrm{Sn} 123 \mathrm{~m}$ & U236 \\
\hline $\mathrm{Be} 7$ & Eu149 & $\mathrm{Pa} 231$ & $\mathrm{Pu} 243$ & Sn 125 & $\mathrm{U} 237$ \\
\hline $\mathrm{Bi} 210$ & Eu152 & $\mathrm{Pa} 233$ & $\mathrm{Pu} 244$ & Sn126 & $\mathrm{U} 238$ \\
\hline $\mathrm{Bi} 211$ & Eu154 & $\mathrm{Pa} 234$ & Ra223 & Sn127 & $\mathrm{U} 240$ \\
\hline $\mathrm{Bi} 212$ & Eu155 & $\mathrm{Pa} 234 \mathrm{~m}$ & $\mathrm{Ra} 224$ & Sr85 & $\mathrm{Xe} 127$ \\
\hline $\mathrm{Bi} 213$ & Eu156 & $\mathrm{Pb} 209$ & Ra225 & Sr89 & $\mathrm{Xe} 131 \mathrm{~m}$ \\
\hline $\mathrm{Bi} 214$ & Fr221 & $\mathrm{Pb} 210$ & Ra226 & Sr90 & Y88 \\
\hline $\mathrm{Bk} 249$ & Fr223 & $\mathrm{Pb} 211$ & $\mathrm{Rb} 83$ & Tb157 & Y89m \\
\hline $\mathrm{Bk} 250$ & Gd151 & $\mathrm{Pb} 212$ & $\mathrm{Rb} 84$ & Tb158 & Y90 \\
\hline $\mathrm{C} 14$ & Gd153 & $\mathrm{Pb} 214$ & $\mathrm{Rb} 86$ & Tb160 & Y91 \\
\hline Cd109 & $\mathrm{H} 3$ & $\operatorname{Pd} 103$ & $\mathrm{Rb} 87$ & Tc97m & Zr88 \\
\hline $\mathrm{Cd} 111 \mathrm{~m}$ & Ho163 & $\operatorname{Pd} 107$ & Rh101 & Tc98 & $\mathrm{Zr93}$ \\
\hline $\mathrm{Cd} 113 \mathrm{~m}$ & Ho166 & $\operatorname{Pd} 112$ & Rh102 & Tc99 & Zr95 \\
\hline $\mathrm{Cd} 115 \mathrm{~m}$ & Ho166m & Pm144 & $\mathrm{Rh} 102 \mathrm{~m}$ & Te121 & \\
\hline Ce139 & $\mathrm{I} 125$ & Pm145 & $\mathrm{Rh} 103 \mathrm{~m}$ & $\mathrm{Te} 121 \mathrm{~m}$ & \\
\hline Ce141 & I129 & Pm146 & Rh106 & Te123m & \\
\hline Ce144 & I131 & Pm147 & Rh106m & $\mathrm{Te} 125 \mathrm{~m}$ & \\
\hline
\end{tabular}



Appendix B

\section{MATLAB GUI Implementation}





\section{Appendix B}

\section{MATLAB GUI Implementation}

The following screen shots show the GUI operation. First, data must be loaded into the GUI. This uses the typical "open" window to browse for a file on the computer (not shown). After a .mat file has been selected, if multiple variables (matrices) are included in the file, then a selection utility appears to select the appropriate file. After data has been loaded, the "Evaluate" button becomes active. This button will characterize each of the spectra contained in the selected data file. Once the fuel spectra have been characterized, the "View Next" and "Save Results" buttons become active. These buttons allow the user to scroll through the results display and save the results to a mat file in the current path named "fuelCharResultsMMDDYYYY" with the current date in the file name, respectively. The file fuelCharGUI.fig is needed to run the GUI, as well as the code given below the figures.
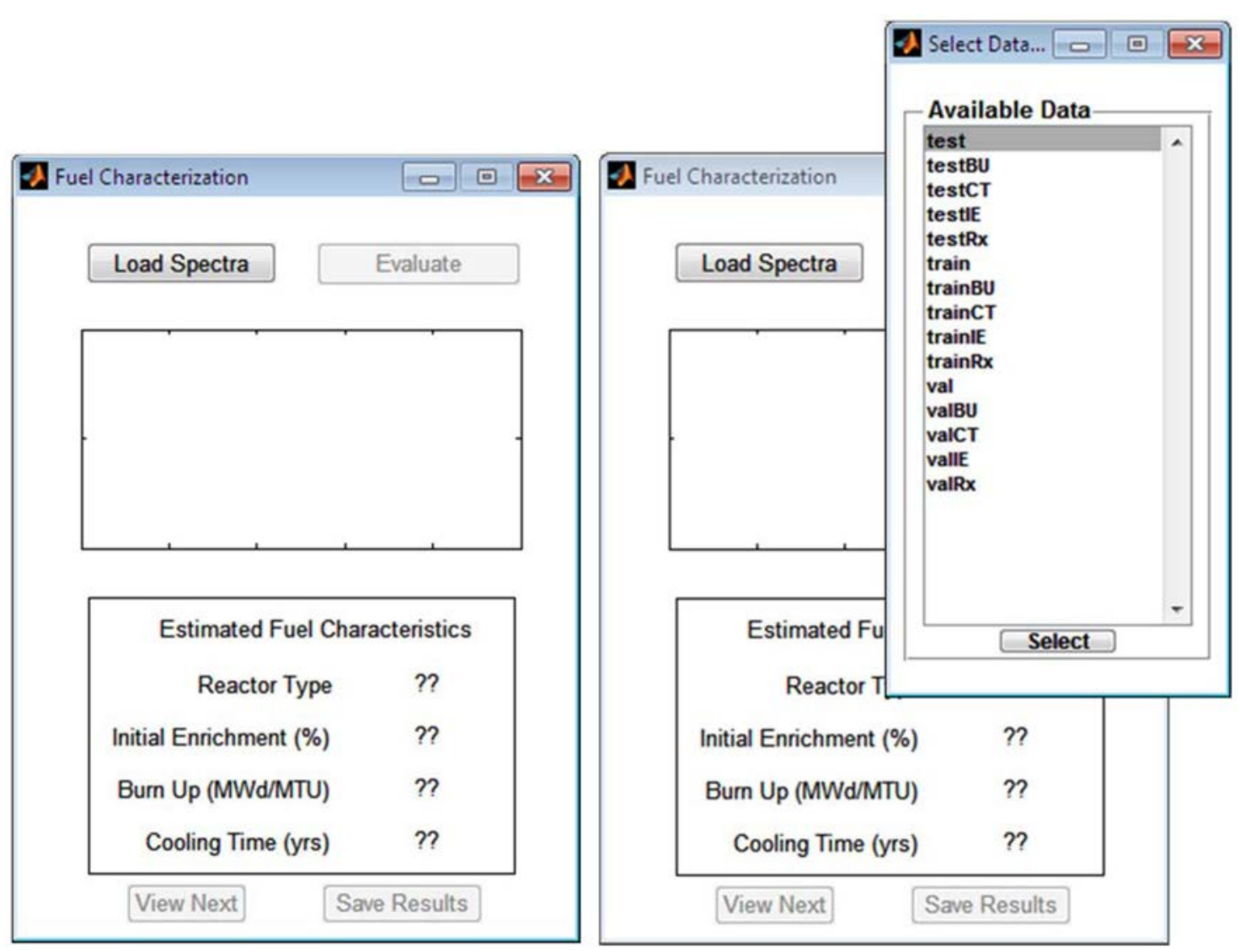

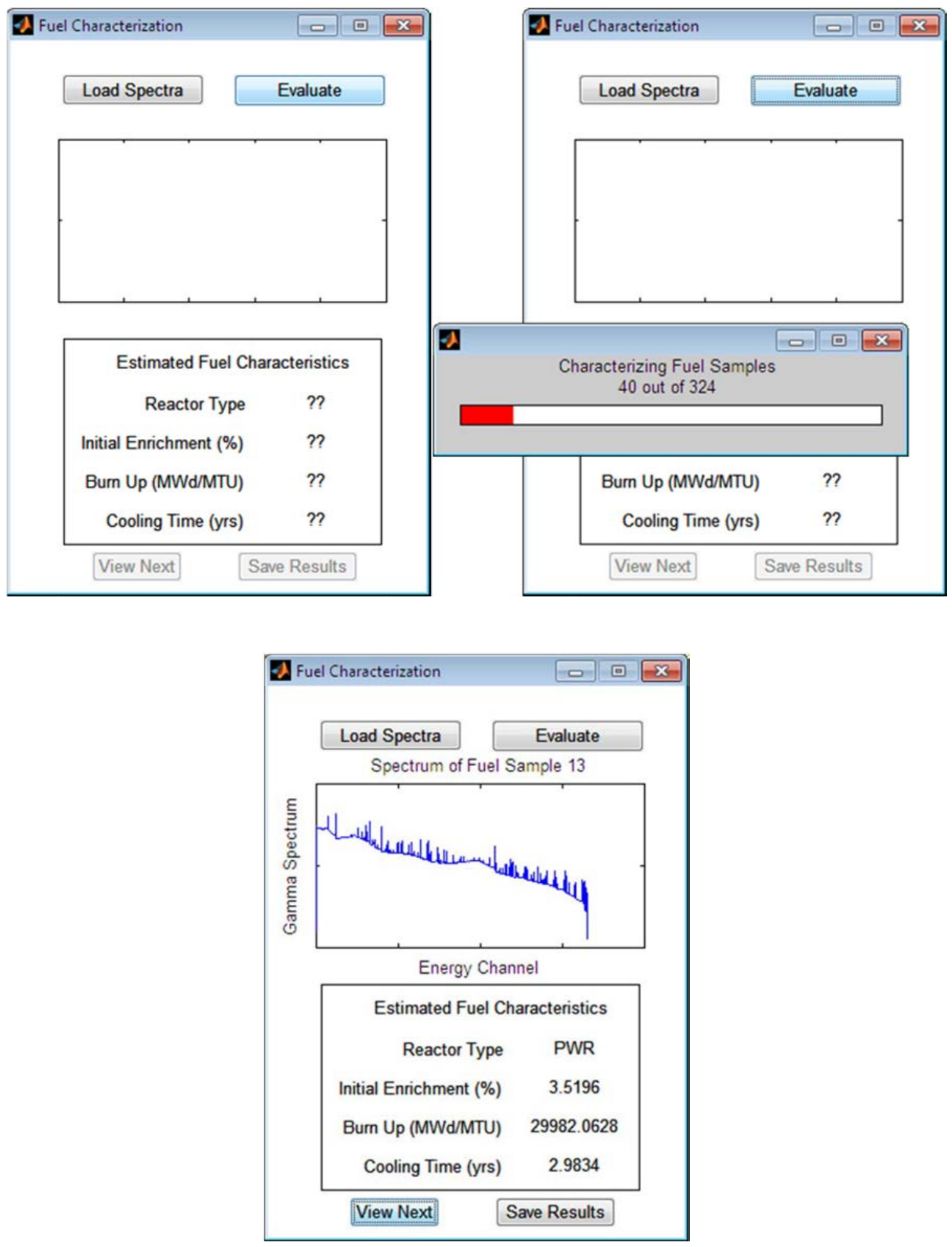



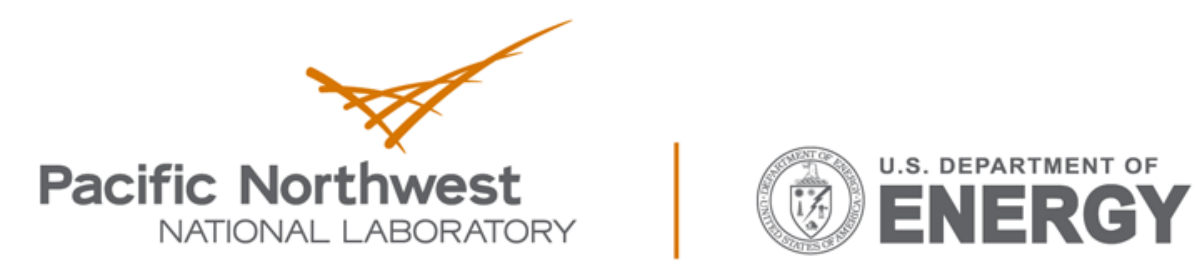

Proudly Operated by Battelle Since 1965

902 Battelle Boulevard

P.O. Box 999

Richland, WA 99352

1-888-375-PNNL (7665)

www.pnnl.gov 